\title{
From concept to delivery: a new tool for courseware developers
}

\author{
H. Freeman \\ S. Ryan \\ De Montfort University \\ Leicester \\ United Kingdom
}

\begin{abstract}
This paper identifies some common difficulties and inefficiencies within the process of courseware development, in particular the conversion of subject domain knowledge into pedagogical sequence. It then describes a suite of tools designed to facilitate the development and delivery of courseware within an academic context.

These tools are designed to support collaborative working among all members of the courseware team. The first and most important tool is the concept mapper. Here the basic structure of the course is defined, the relationships between elements is formalized and represented graphically, the textual content is written and other multimedia resources described. The two remaining tools of the suite convert the map into a working multimedia deliverable.
\end{abstract}

Main conference themes: methodologies, software, tutoring

Educational areas: higher education

Study topics:

Secondary keywords: authoring systems, courseware, knowledge representation, modelling, multimedia, teaching methods 


\section{INTRODUCTION}

The ideas and issues behind the tools described in this paper came from our experience in developing courseware within our own institution, De Montfort University, and from an European Commission (EC) funded project to produce courseware with partners in Italy and Greece.

The single issue closest to the centre of the problem is that of communication, or rather its lack. In the EC project the problems caused by physical separation between members of the courseware team were compounded by their ideological and experiential separation. For example, courseware designers and programmers both accept the notion of a cyclical process of development, yet the component phases and their functions could not easily be mapped to a common model.

Our evaluation of the EC project and subsequent research caused us to focus on the conceptualization and mapping of a courseware project as most in need of development and support. A tool was developed whose function was to enable and enhance communication between team members regarding the knowledge structures, pedagogical flows and courseware resources such as graphics and sound. The tool, ConceptMapper, enabled collaborative working, and provided maximum flexibility for customization, change and updating during both the development and delivery cycles.

Most concept mapping tools have one function only. However, the tool described here works in tandem with, and sends information to, two further tools. In this way, resources (graphics, sound and text) and processes (links and sequences) may be linked to their definitions in the concept map by an editor tool. Also a final page description may be produced which is converted into a CAL package by the third tool, the Runner.

\section{Courseware development tools}

The use of concept maps and semantic networks for the clarification of issues or as aids to learning is not new. In 1974 the BBC Further Education Advisory Council published a book [1] to accompany a programme called 'Use Your Head' which advocated the use of concept maps. Rowntree [2] proposes the use of concept maps in the analysis phase of the preparation of open, distance and flexible learning materials. The rapid drop in price and increase in performance of personal computers has stimulated the development of hypertext systems which have at their core, either explicit (i.e. hard coded) or implicit (i.e. index driven), concept maps. Whilst hypertext has been seen largely as a tool for managing and presenting information, recent research has focused on the representational capabilities of the hypertext data model, e.g. [3, 
4], as an agent for knowledge structuring within the process of instructional design.

Two of the implicit processes in developing instructional materials are on the one hand the use of knowledge structures to organize and categorize content, and on the other hand the development over time of sequences or schemes which describe the paths a learner might take through a knowledge domain.

The tools described here attempt to make these processes explicit and available to all players in the development of courseware materials. Because this is not an individual effort it is important that the skills of the specialists involved be pooled and focused on issues as diverse as describing knowledge structure and content, selecting teaching strategies and producing uniform screen designs. Even where courseware design is attempted by individuals, as is becoming sadly commoner, the tools constrain the user within a productive yet flexible environment which offers access to all phases of the courseware development life cycle.

Many concept mapping tools, such as the Designer's Notepad [5], are designed to support the early stages of software design, such as brainstorming, refinement of ideas and the consideration and selection between alternative design options. The suite of tools described here advances this approach, being closer to Rada's OSCAR [6], a model based system which uses a concept map structure to enable coordination, collaboration, definition and production of courseware.

Our aims in designing the suite were to enable clarity of purpose and communication, and facilitate collaboration between the different parties involved in the life cycle phases of analysis, design, development, implementation and evaluation.

Modern icon based authoring systems offer many advantages to the developer but there is a danger that systems tend to encourage the collapse of the various roles required for development into one person-the universal courseware expert. When using this system there is a tendency not only to map out the courseware, but in the act of mapping it out, to try to create it simultaneously. At its most extreme 'the universal courseware expert' creates 'screen one', produces all text and graphics for it, links it to 'screen two', produces the text and graphics and so on. In effect, what happens is that the producer is attempting to structure and plan the courseware and produce it simultaneously.

We have attempted to develop an icon based concept mapping tool which will allow the subject expert and instructional designer to map out and plan the entire piece of courseware project before the courseware is created. Using the system the developer may, via the manipulation of icons, try out the different 
links and routes, add and delete proposed sections and run through various sequences without being concerned about the actual delivery. This encourages the top-down planning of the entire courseware prior to any attempt to construct it. The act of planning and structuring is therefore separated from the production.

\section{Integrating planning and development}

But while separating out the process of planning, we also wish to re-integrate it. If a map of ideas alone were being provided, this could be done on paper or by using commercially available concept mapping packages. Part of the planning process is to consider how the ideas mapped out will be realized and delivered via various media, and how the features of the media can best be used for teaching purposes. Therefore, as part of the conceptualization, it is necessary to specify how graphics, text, video or sound will be used and incorporated in the delivery system.

The concept mapping stage provides a way of indicating how media and content will be integrated within the final structure which information serves as a template for others to build and implement in the delivery system.

\section{Team roles}

The tools provide ways in which each team member can clearly define the own role within the overall structure. The process facilitates project management. A graphic designer for example may examine the concept map which will indicate the ideas for graphics the author suggests should accompany particular sections, what menus are required, and their location. This provides the designer with a template in which to work and interact with other team members.

\section{The need for flexibility}

In developing the generalized multimedia shell a key consideration was to produce a development and delivery system which would be as flexible as possible. In this context flexibility has three elements:

- the ability to make changes quickly and easily while the courseware is being developed;

- the ability to update and change elements once the courseware has been produced; and

- to facilitate cross platform transfer. 


\section{Changing while the courseware is being developed}

The philosophy underpinning the Generalised Multimedia Shell assumes that during courseware development many changes will be made to the system. Such changes do not in any way represent the fault or failing on any team member's part, rather are a natural element of the process of courseware development. We therefore wish to be able to change as many elements as possible at any time up to and including the final version without having to rebuild or recompile the system.

\section{Updating}

Most courseware will often need frequent updating separate from debugging. Content in an academic context is liable to change and require fresh and different input, perhaps as a result of student feedback or because the material is becoming dated. Good updating facilities are particularly important given the relatively high development costs of courseware.

\section{Transfer implications}

The shell should work across platforms. The initial version runs on the Macintosh platform, a Windows version will be available in early 1995. All materials and resources which are generated for the Macintosh version, should be usable on other platforms with a minimum of conversion problems.

This does not necessarily imply two separate software suites. The final courseware is built up in the delivery shell from descriptions passed from the editor and the concept mapper in the form of text files, and resources are held externally in standard file formats. Therefore it should be possible to develop the structure entirely on the Macintosh and then run it from a Windows based delivery shell having where necessary converted the standard format graphics, video or animation files using commercially available file translation utilities.

\section{Achieving flexibility}

The key to flexibility described was the use of programming techniques which call in resources (text, sound, video, animation etc.) as they are required, with resources stored in specified directories in standard formats. All text files for example are held as ASCII files which can be modified and changed using any standard word processor. All graphic elements are stored as PICT files which can be produced and manipulated in any standard graphics package or grabbed from a video source using common digitizing cards or Apple's built-in AV technology.

All resources therefore can be built up and inspected outside the shell, offering the possibility of subcontracting the production of graphic or digital video elements. The most appropriate tools can therefore be chosen for each 
task. Graphics can be produced in high specification graphics packages, rather than using an authoring system's own tools. Video and animation sequences can be produced and modified in the most appropriate packages.

\section{Existing materials can also be easily incorporated}

Resources can be modified or replaced at any point during development, and when it is necessary to update, the modified files can be substituted for the originals without altering the structure.

The second way in which flexibility is achieved rests upon the way the delivery system works. To the user the application may appear to be made up of a series of linked screens, but in practise each screen is assembled from a series of discrete elements such as the menu, title bar, text field, video area, etc. The description of these elements is held in a text file. Thus the appearance of the screen can be changed by adding or deleting different items in this text file which are then called by the application the next time it is run.

\section{PROPERTIES AND USAGE OF THE SOFTWARE SUITE}

The suite consists of three tools, ConceptMapper, Editor and Delivery Shell.

\section{The ConceptMapper}

The ConceptMapper handles knowledge structures, pedagogical flows and courseware resources.

On opening, the user is presented with a large work area, (equivalent to six landscape A4 pages, but expandable to 38 feet by 38 feet) and a floating control palette. The user creates 'nodes' which represent components of the knowledge domain. The nodes may be linked to describe the relationship between them. Figure 1 shows part of a typical example.

New nodes may be created, deleted, moved, linked, unlinked and relinked as necessary. In the courseware development lifecycle initially the nodes (and the concept map they make up) represent the knowledge domain of the subject expert(s). By double clicking a node a new window opens into which text may be typed/imported, and other processes performed. Eventually the map becomes a pedagogical structure through the interaction of subject experts, educational designers and the sequence definition process of the conceptmapper tool. In this stage the relationship between nodes is defined in terms of menu and sequence.

Thus the map evolves from a description of the knowledge domain as seen from the point of view of the subject expert(s) into a description of pedagogical flow, by the definition of a node either as a menu node or a sequence node. 


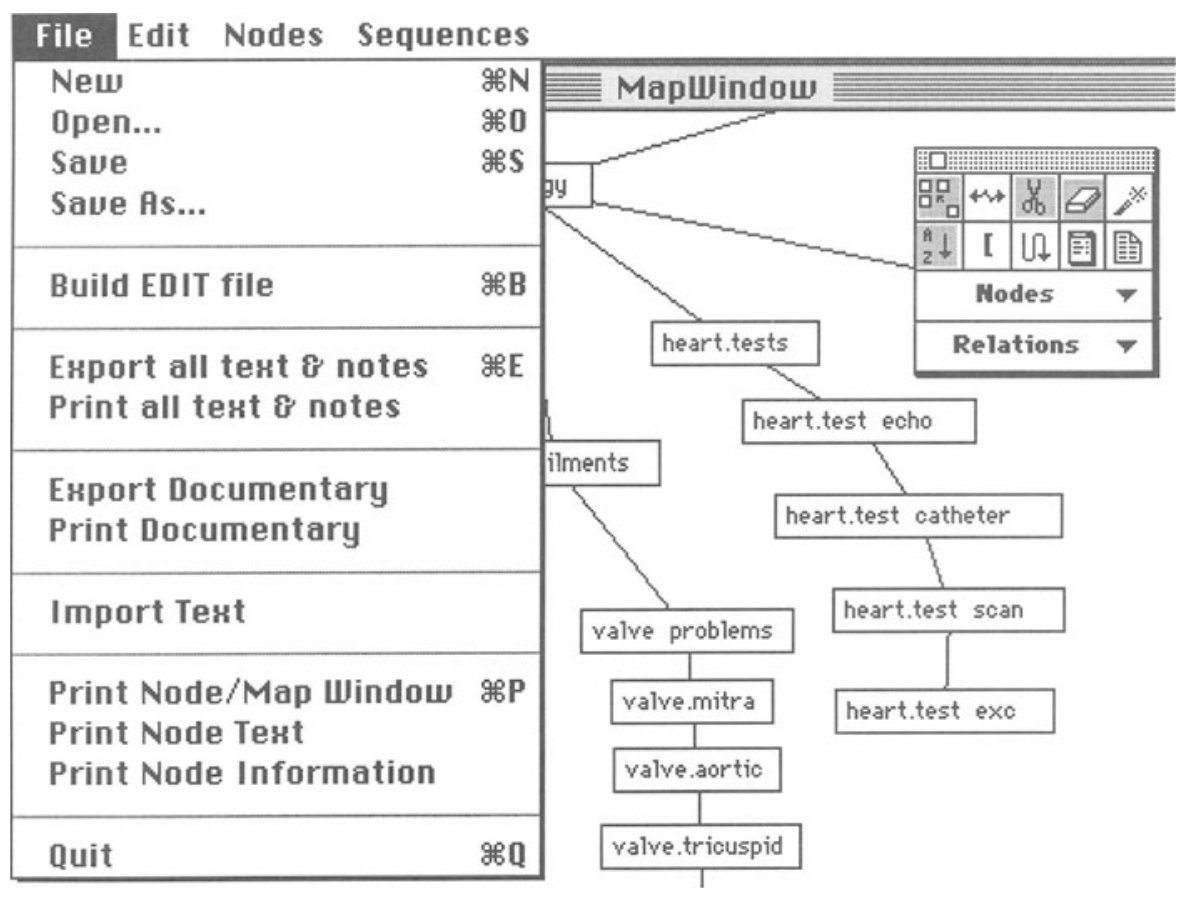

Fig. 1. The concept mapping window

Menu nodes are decision points for users, and offer the choice of many destinations: sequence nodes only offer the ability to go to prior or next nodes, or the last branch (the last menu node). Thus the map changes its function through time.

When a node is double clicked a new window is opened containing a text file with the indicative content and details of pictures, graphics and sound. Other elements to be included can be logged. This is done either in the form of notes describing the particular feature or via the use of flags to indicate hotspots in the text field where user mouse clicking will bring in other resources. See Figure 2.

Note the flags in Figure 2 indicating where glossary and graphics are to be imported into the text field and also the descriptor of what will happen when the screen is entered by the user. The word 'loopers' has been flagged, meaning that the subject expert/courseware developer considers this word of interest to the end user. The resource at this point has not yet to be available, it is merely an indication to all other parties involved that it might be included. 


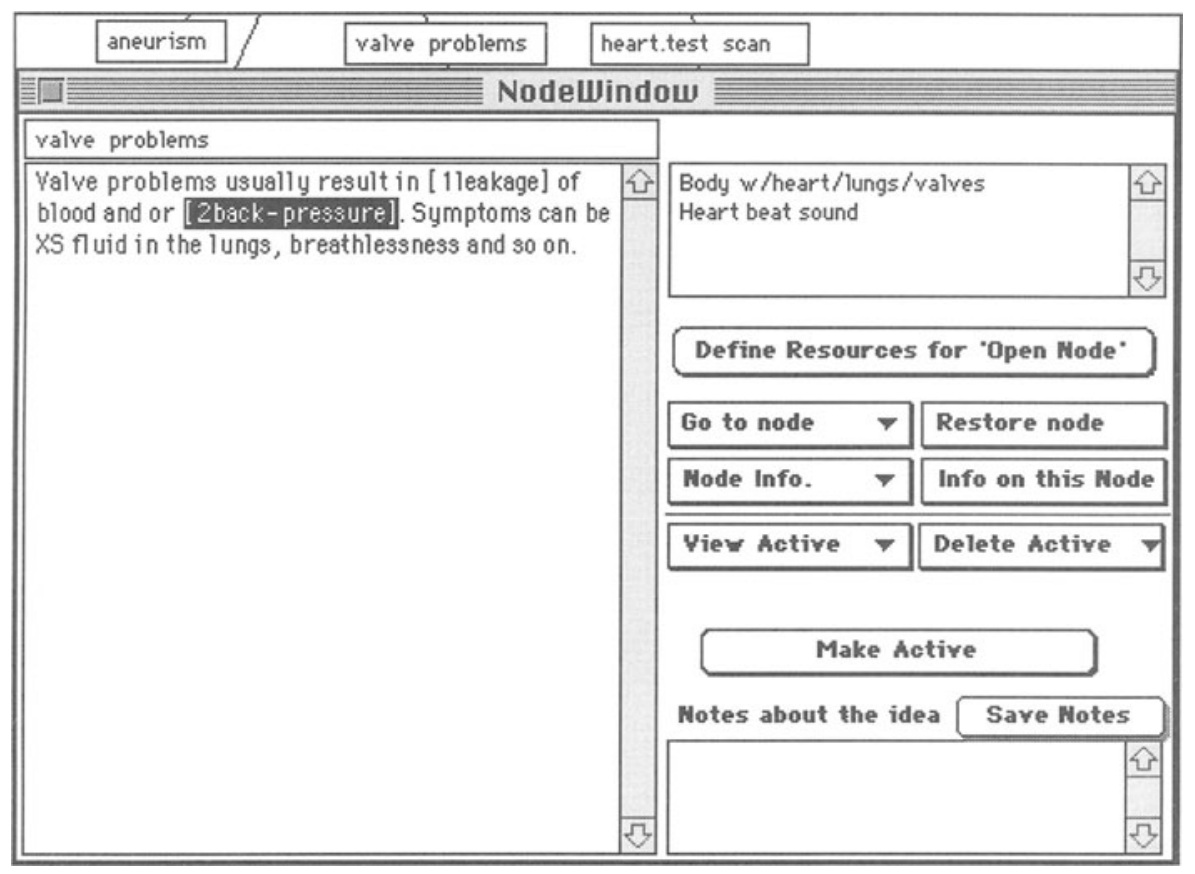

Fig. 2 The concept mapper descriptor window

The production of the resource will be done later by a graphic designer, video engineer, subject specialist or other person with specific skills: the subject expert is insulated from the desire to produce the resource NOW.

The information associated with the nodes may be printed out and provided to other members of the team as a hard copy template for working on. In addition the information is exported to the editor for assembly into the final delivery system. Also the sequence of movement between the individual nodes is defined and exported to the editor.

\section{The Editor}

The final delivery product consists of:

- a delivery shell with a directory containing resources (with subdirectories of text files, graphics files, movies and sound files);

- the 'project' file, an ASCII file which controls the order of movement between the nodes, the nature of the resources used at each node, and the possibilities for action by the user. 


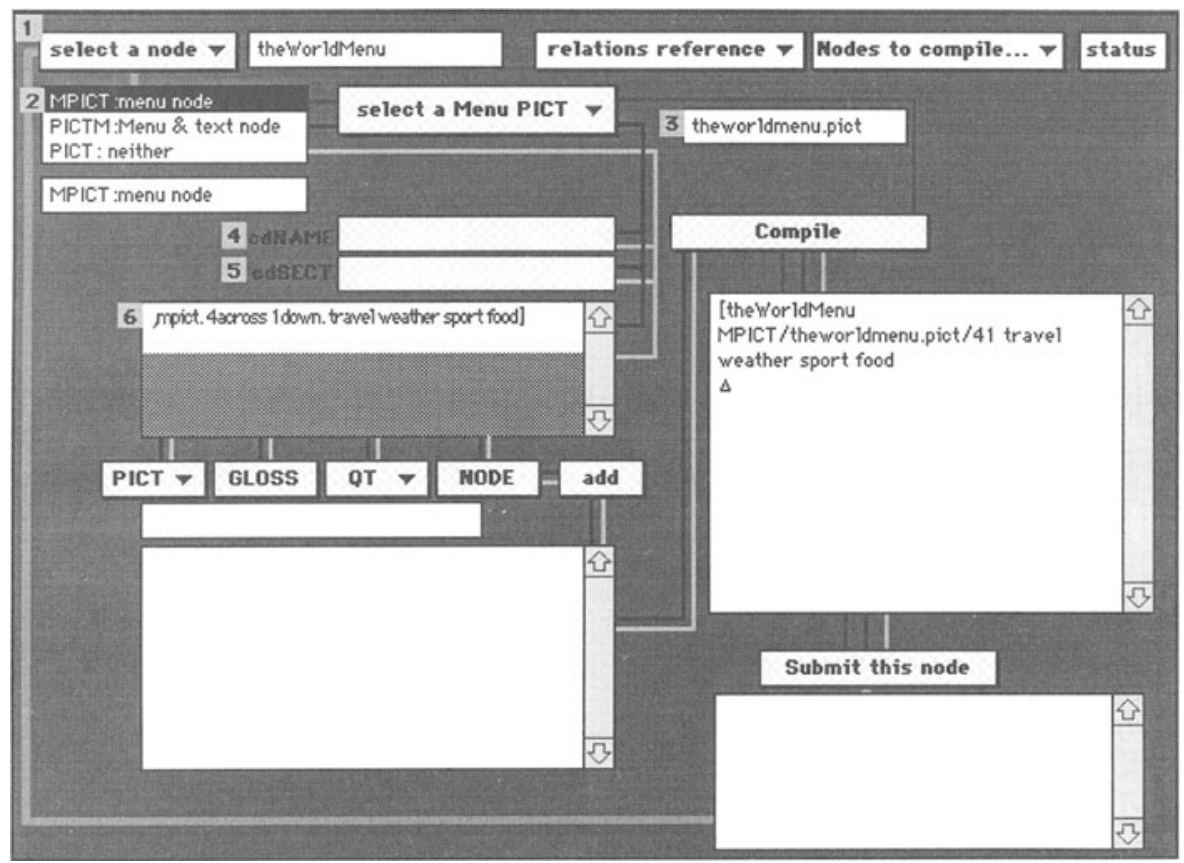

Fig. 3 An example of an editor screen

Essentially the editor works by taking a node from the list of nodes created in the concept mapper and requesting the user to identify the resources to be associated with each of the components such as flagged words, menus and so on. Here text files can be viewed and amended, QuickTime movies and graphics files viewed and sound files heard. This information is then compiled into the Datafield for final delivery.

The editor provides the opportunity for the original ideas and concepts of the subject specialists to be refined and appropriate resources linked. There is also opportunity for changing the sequencing initially identified in the concept mapping section.

\section{The Delivery Shell}

The delivery shell reads in the project file created in the concept mapper and editor describing the sequencing and resources needed for the running of the courseware. A diagrammatic version of the concept map is rebuilt in the delivery shell at runtime and identifies the nature of each node and the relationships it bears with others by means of colour and shape. Moving the 
mouse across the nodes in the map causes a brief summary of the node to appear, and clicking on the map node causes a direct jump to that node. A more traditional text based indented menu structure is also provided: the method used depends on the target group.

If there are problems-perhaps a resource has been misidentified-there is a facility to modify and change both the Infofield and Datafield originally created in the two previous sections.

\section{Advantages of the system}

What are the main advantages of the system? The key advantages are prioritization of the conceptualization and overall design, and secondly the flexibility of holding resources in standard file formats external to the application. A third advantage is the management of the production process.

The combination of the concept mapper, the editor and the delivery shell makes the processes of development more transparent. A graphic designer for example can see the entire shape of the project from the concept mapper and develop graphics in that context. The designer comments on the form and structure and the interaction between the elements is facilitated the tools.

The project manager can identify at an early stage what video, graphic or other elements are going to be needed, and when, and plan resources accordingly on the basis of a resource list print out by the editor.

The subject specialist can see how the original plan is being realized and can make or suggest modifications at any stage.

An instructional designer can build up a record of maps of various projects and in staff development show how courseware is built up and developed.

\section{CONCLUSION}

The generalized multimedia shell is a suite of tools designed to facilitate the development of courseware. The tools emphasize concept mapping and structuring prior to building the courseware and allow the conceptual structure to be related to the resources required for implementing this structure.

Because the resources are held externally, maximum flexibility results and the use of standard graphics, movie and text producing packages can be optimized. Although the shell inevitably has limitations and restrictions, its open ended structure allows further development and expansion. The authors are planing future developments on the basis of evaluation. Evaluation copies are available in return for a structured assessment and description of usage. 


\section{REFERENCES}

1. Buzan, T. (1974) Use Your Head. BBC Further Education Advisory Council.

2. Rowntree, D. (1994) Educational technology in curriculum development. Harper and Row, London.

3. Marshall, C., Halasz, F., Rogers, R. and Janssen, W. (1991) Aquanet: a hypertext tool to hold your knowledge in place. Proceedings of Hypertext 91, pp. 261-275. ACM.

4. Russell, D.M., Moran, T.P. and Jordan, D.S. (1987) The Instructional Design Environment, in Intelligent Tutoring Systems: lessons to be learnt. Psotka, J., Massey, L.D. and Butler, S.A. (eds).

5. Twidale, M.B., Rodden, T. and Sommerville, I. (1994) The use of a computational tool to support the refinement of ideas. Computers in Education $22(1 / 2)$ pp. 107-118.

6. Rada, R., Deakin, A.G. and Beer, M.D. (1993) Collaborative development of courseware: part one-examples. Intelligent Tutoring Media 4 (2). 